\title{
ORIGINAL ARTICLE Beneficial effects of early hemostasis on spinal cord injury in the rat
}

Updated online 21 June 2016: This article was originally published under NPG's License to Publish, but has now been made available under a CC BY-NC-SA 4.0 license. The PDF and HTML versions of the paper have been modified accordingly.

\section{H Fan, K Chen, L Duan, Y-Z Wang and G Ju}

\author{
Study design: Experimental study. \\ Objectives: To investigate the effect of early hemostasis on spinal cord injury (SCI). \\ Setting: Fourth Military Medical University, Xi'an, China.
}

Methods: Sprague Dawley rats were used. Hematoxylin and eosin (HE) staining was performed to observe hemorrhage at different time points $(2,6,12,24$ and $48 \mathrm{~h}$ ) after $\mathrm{SCl}$ to determine the time window of hemostatic drug administration ( $n=3$ per time point). Three different concentrations of Etamsylate $\left(0.025,0.05\right.$ and $0.1 \mathrm{~g} \mathrm{~kg}^{-1}$ ) were administered immediately and 5 and $10 \mathrm{~h}$ after $\mathrm{SCl}$ to evaluate the effective dosage ( $n=6$ per group). Another 82 rats were then randomly divided into two groups, Etamsylate group $\left(0.1 \mathrm{~g} \mathrm{~kg}^{-1}, n=41\right)$ and glucose control group $(n=41)$. Nissl staining was performed to observe neurons at 10 days post injury. Immunohistochemistry, western blot and quantitative real-time PCR were performed to detect tissue necrosis at 7 d.p.i., the activation of astrocytes and microglia/macrophages and lesion cavity at 10 d.p.i. Basso-Beattie-Bresnahan scoring and rump height index assay were used to examine locomotion recovery.

Results: Early hemostasis reduced the lesion area and tissue necrosis, enhanced neuronal survival, alleviated the activation of microglia/macrophages and astrocytes and facilitated functional recovery after spinal cord contusion in rats. Early hemostasis decreased hemorrhage area and lesion area after spinal cord transection in rats.

Conclusion: The present study demonstrated that early hemostasis has beneficial effects on SCl in the rat. It has the potential to be translated into clinical practice.

Spinal Cord (2016) 54, 924-932; doi:10.1038/sc.2016.58; published online 3 May 2016

\section{INTRODUCTION}

It has been estimated that in North America, $~ 39$ people per million suffer from spinal cord injury (SCI) each year, and in Western Europe the incidence is 16 per million. ${ }^{1}$ People who suffer from severe SCI with paralysis of a part of their bodies may cause a heavy burden to themselves, their family as well as the society. Furthermore, their life expectance is about the same as that of healthy people. Therefore, the study of SCI is a major project the world over.

After SCI, either contusion or transection, there is always immediate hemorrhage leading to subsequent death of neurons and oligodendrocytes. $^{2-5}$ Hemorrhage also accounts for activation of microglia by components derived from blood. ${ }^{6-8}$ The iron released by lysis of red blood cells (RBCs) plays an important role in polarizing the pro-inflammatory M1 phenotype of microglia that is detrimental. ${ }^{9}$

It has been demonstrated in recent years with computer tomography and magnetic resonance imaging that substantial parenchymal hemorrhage lasts for several hours in patients after SCI, ${ }^{10,11}$ leaving enough time for hemostasis. In the light of the above-mentioned pathological changes after SCI, we therefore investigated early hemostasis in a rat model.

Etamsylate, also named Ethamsylate, which acts on the first step of hemostasis by improving platelet adhesiveness and restoring capillary resistance, ${ }^{12,13}$ has been effectively and safely used in clinical cases such as menorrhagia, periventricular hemorrhage and postsurgical bleeding without severe side effect, except it may cause mild skin allergy. ${ }^{12,14}$ Blood flow changes should be considered when hemostatic treatment was used. No influence of blood flow was observed after Etamsylate administration in previous studies. ${ }^{15,16}$ That is why we choose Etamsylate for early hemostasis to treat SCI.

Although the incidence of transection of spinal cord is much lower than that of contusion, there is much more perfuse bleeding in transection. Therefore, both were included in our study of early hemostasis. As hemostasis will cause no harm, it can be easily translated to clinical application and be beneficial for the patients.

\section{MATERIALS AND METHODS}

\section{Animal models}

A total of 101 male Sprague Dawley rats (200-250 g) were purchased from the Laboratory Animal Center of the Fourth Military Medical University (Xi'an, China). All the animal experiments were approved by the animal care and use committee of the Fourth Military Medical University. Animals were anesthetized with $1 \%$ sodium pentobarbital $\left(60 \mathrm{mg} \mathrm{kg}^{-1}\right)$, followed by bilateral laminectomy of vertebrae T8-T9. For spinal cord contusion (SCC), Tetzlaff's spinal cord lateral crush model ${ }^{17}$ was followed and modified to improve the consistency of the experimental data as we described previously. ${ }^{18}$ Tetzlaff used 
a pair of forceps to manually compress the spinal cord from the lateral sides. It is difficult to ensure the compression completes right at the midline of the spinal cord. It may either deviate to the right or left of the midline. We therefore designed a mechanical device. We first close the tow plates of the forceps (53327T, 66 Vision-Tech Co., Ltd, Suzhou, China), lower it down and adjust its tips to align with the midline of the cord. The tips are then widened and lowered along both sides of the dura mater of the spinal cord till the tips touch the bottom of the spinal canal. With our device it is much easier and more accurate to close the forceps at the set parameters, such as the speed, distance between the tips, time to remain the closed condition and the speed of releasing the closure. Thus, the injury of the spinal cord made in different animals is comparable because the spinal canal is round in shape, the lowering of the tips of forceps stops at a distance from the midline, and the remaining space of the spinal canal in each case may not be the same. In the present study, the width between the forceps tips was set at $0.5 \mathrm{~mm}$ when fully closed and the crush remained for $20 \mathrm{~s}$. Manual bladder expression was performed once a day after surgery.
For spinal cord transection (SCT), the T8 vertebral plate was removed, and the dura mater cut open with a pair of iridectomy scissors from the midline to the ventral half of the broken vertebral pedicle. To avoid the midline blood vessels, the left half of the spinal cord was transected with a surgical blade down for $1.5 \mathrm{~mm}$, starting from $0.5 \mathrm{~mm}$ lateral to the midline of the spinal cord.

Hemostatic drug and its administration

Etamsylate (Shan Dong Fang Ming Pharmaceutical Group Ltd, Heze, China) was diluted with $10 \%$ glucose. The dosage in rats is calculated by the percentage of weight of rat by that of adult human. Etamsylate was administrated via tail vein injection of $0.025,0.05$ and $0.1 \mathrm{~g} \mathrm{~kg}^{-1}$ t.i.d. (three times per day), immediately and 5 and $10 \mathrm{~h}$ after SCI. As the control, $10 \%$ of glucose was used. For evaluating the effects of early hemostasis on SCT in rats, $0.1 \mathrm{~g} \mathrm{~kg}^{-1}$ of Etamsylate was administered at the same time points after surgery. The experimental design is shown by the flow diagrams (Figure 1). The model of $\mathrm{SCI}$ is a severe trauma to rats. For adhering to the rules of 3Rs (Replacement, Refinement and Reduction of animals in research), small sample size of animals

a HE Staining for hemorrhage detection( $n=3 /$ time point $)$

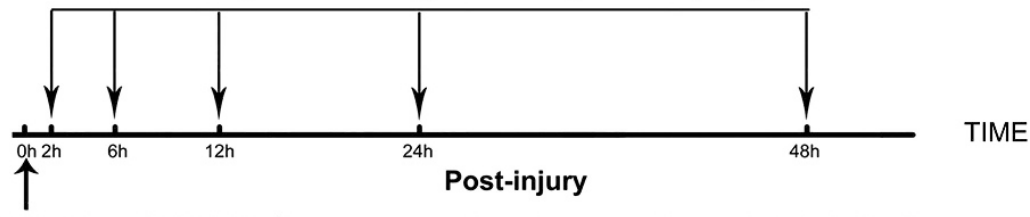

SPINAL CORD CONTUSION (To determine time window of drug administration)

b

Tail vein injection: Glucose,

$0.025 \mathrm{~g} / \mathrm{kg}, 0.05 \mathrm{~g} / \mathrm{kg}, 0.1 \mathrm{~g} / \mathrm{kg}$ of Etamsylate $(\mathrm{n}=6 / \mathrm{group})$

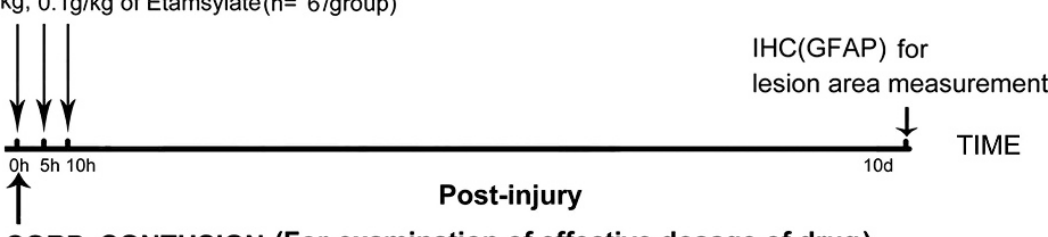

SPINAL CORD CONTUSION (For examination of effective dosage of drug)

C

Behavior training Tail vein injection: Glucose,

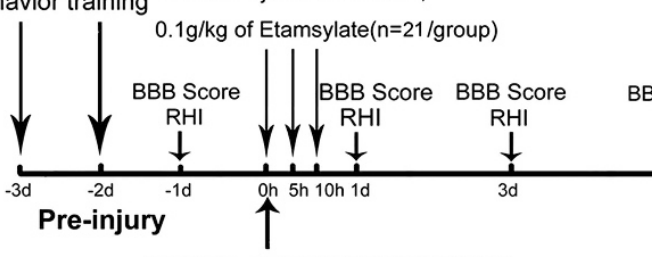

SPINAL CORD CONTUSION

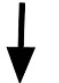

Nissl staining( $n=6 /$ group)

IHC, qPCR and Western

blot(n=3/group) for necrosis

detection(RIP3 and MLKL)

BBB Score

$\mathrm{RHI}$
$\mathrm{IHC}(\mathrm{n}=6 /$ group $), \mathrm{qPCR}(\mathrm{n}=3 /$ group $)$ and

Western blot(n=3/group) for glial

activation detection(GFAP and Iba-1)

BBB Score

$\mathrm{RHI}(\mathrm{n}=6 /$ group)

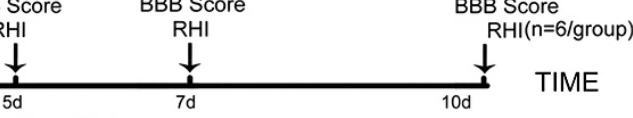

Post-injury

1

d

Tail vein injection: Glucose,

$0.1 \mathrm{~g} / \mathrm{kg}$ of Etamsylate ( $\mathrm{n}=10 / \mathrm{group}$ )

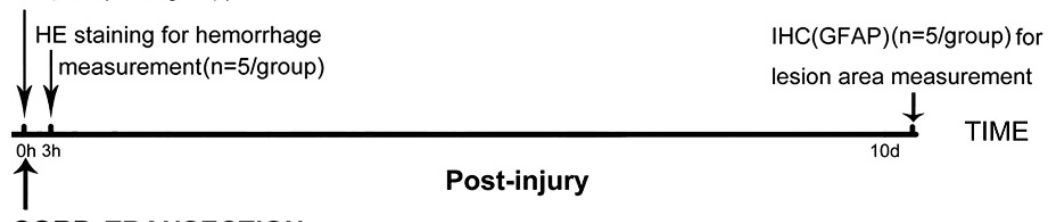

SPINAL CORD TRANSECTION

Figure 1 Flow diagrams showing the experimental design. (a) Experiment for hemorrhage detection to determine the time window of drug administration. (b) Dose-response experiment for examination of effective dosage of Etamsylate. (c) Main experiment of the effects of Etamsylate on ScC. (d) Experiment of the effects of Etamsylate on SCT. 
was used. Six, five and three rats per group were used in different studies. Six rats per group were used for the detection of lesion area, locomotion improvement and numbers of survived neurons in the SCC. Five rats per group were used to evaluate the effect of Etamsylate on SCT. The study of protein expression alongside the corresponding mRNA levels of RIP3 (receptor-interacting serine-threonine kinase 3), MLKL (mixed lineage kinase domain-like protein), Iba-1 (ionized calcium-binding adapter molecule 1) and GFAP (glial fibrillary acidic protein) by western blotting, immunohistochemistry and quantitative real-time PCR were performed by using three rats per group, and the results from these complementary experiments showed significant differences and are consistent with each other, and therefore additional animals were not added.

\section{Fixation and section preparation}

The animals were anesthetized and perfused intracardially with $200 \mathrm{ml}$ of saline followed by $400 \mathrm{ml} \mathrm{4 \%}$ paraformaldehyde. Subsequently, $2 \mathrm{~cm}$ spinal cord segments containing the lesion site were removed and immersed in $25 \%$ sucrose at $4{ }^{\circ} \mathrm{C}$ until they sank. Eight sets of serial sagittal sections ( $10 \mu \mathrm{m}$ thick) from contusive cord or of serial horizontal sections from transected spinal cord (10 $\mu \mathrm{m}$ thick) were prepared on a cryostat. The thickness of the section ensures that the plane of central canal is cut through on every slide, rendering random selection of the slide possible.

\section{Hematoxylin and eosin (HE) staining}

The sections were rinsed in distilled water and were then put into hematoxylin solution for $3 \mathrm{~min}$, followed by differentiation in 10\% acid alcohol for $15 \mathrm{~s}$. After washing with tap water for $30 \mathrm{~min}$, the sections were dehydrated through 70,80 , 95, and 100\% alcohol for $2 \mathrm{~min}$ each, and then were stained with eosin for $1 \mathrm{~s}$.

\section{Nissl staining}

To assess the survival of neurons, the sections were subjected to Nissl staining. The sections were immersed in $1 \%$ thionine solution at $50^{\circ} \mathrm{C}$ for $45 \mathrm{~min}$, and differentiated with $70 \%$ alcohol for $\sim 5 \mathrm{~min}$.

\section{Immunohistochemistry}

The slides were blocked with $0.01 \mathrm{~m}$ phosphate-buffered saline containing $3 \%$ bovine serum albumin and $0.3 \%$ Triton X-100 for $30 \mathrm{~min}$, and were incubated with mouse anti-GFAP (1:500, Millipore, Billerica, MA, USA) for the identification of reactive astrocytes, rabbit anti-Iba-1 (1:1000, Wako, Osaka, Japan) for the identification of microglia/macrophages, rabbit anti-RIP3 (1:200, ENZO, New York, NY, USA) for the identification of necrotic cells or rat antiMLKL (1:200, Millipore) for the identification of necrotic cells at room temperature overnight. After three washings in $0.01 \mathrm{~m}$ phosphate-buffered saline, the sections were incubated with the corresponding secondary antibodies for $4 \mathrm{~h}$ at room temperature in a dark environment. All sections were counterstained with Hoechst 33342 (Sigma, Santa Clara, CA, USA) to identify nuclei.

\section{Western blot}

Spinal cord segments $(1 \mathrm{~cm})$ with the lesion site approximately at the middle were collected, and homogenized with ice-cold lysis buffer containing phosphatase inhibitors and proteinase inhibitors. Equal amounts of protein were separated in 10\% SDS-polyacrylamide gel electrophoresis, and transferred onto polyvinylidene difluoride membrane, followed by blocking the membrane with $5 \%$ nonfat milk for $1 \mathrm{~h}$ at room temperature. Membranes were incubated with primary antibodies for GFAP (1:1000, Millipore), Iba-1 (1:1500, Wako), RIP3 (1:500, ENZO), MLKL (1:500, Millipore) or $\beta$-actin $\left(1: 5000\right.$, Sigma) at $4{ }^{\circ} \mathrm{C}$ overnight. After washing with Tris-buffered saline containing $0.1 \%$ Tween-20, membranes were incubated with corresponding secondary antibody conjugated with horseradish peroxidase (1:8000, Jackson ImmunoResearch, West Grove, PA, USA) for $1 \mathrm{~h}$ at room temperature. Bands were detected with the Bio-Rad Image Lab system (Hercules, CA, USA). ImageJ (NIH, Bethesda, MD, USA) was used for measurement of the densities of specific bands.

\section{Quantitative real-time PCR}

Total RNAs from $1 \mathrm{~cm}$ of the spinal cord segments with the lesion site approximately at the middle were isolated by RNAiso plus (Takara Bio Inc.) according to the manufacturer's instruction at 7 days, and 10 days after SCC. By using the SYBRGreen reaction kit, quantitative real-time PCR was performed (Takara Bio Inc., Kusatsu, Japan) with a Bio-Rad CFX 96 Real-Time System. The specific primers used in this study are as follows: rat GFAP: forward primer, 5'-CCAGATCCGAGAAACCAGCC-3' and reverse primer, 5'-CCG CATCTCCACCGTCTTTA-3'; rat Iba-1: forward primer, 5'-GTCTGAGGA GCTATGAGCCAG-3' and reverse primer, 5'-CTCCATGTACTTCGTCTTG AAGG-3'; rat RIP3: forward primer, 5'-GGAGTCAGGGGAATCAAGCC-3' and reverse primer, $5^{\prime}$-TTTGTAGTCTTTGACCTCTTGTTGG-3'; rat MLKL: forward primer, $5^{\prime}$-TCTGGAACTCGGGGTATGGAT- $3^{\prime}$ and reverse primer, $5^{\prime}$-GCACACGGTTTCTTAGACGC-3'; rat $\beta$-actin: forward primer, $5^{\prime}$-AGATC AAGATCATTGCTCCTCCT- $3^{\prime}$ and reverse primer, 5'-ACGCAGCTCAGT AACAGTCC- $3^{\prime}$.

\section{Evaluation of locomotion}

The Basso-Beattie-Bresnahan (BBB) scale ${ }^{19}$ was used to evaluate locomotion at baseline (1 day before surgery) as well as 1, 3, 5, 7 and 10 days after SCC. Two independent observers who were blinded to the experiments assessed the behavioral scoring.

The rump height index (RHI) assay is to estimate the strength of the animal to support the body weight by the hind limb while walking on a wooden plate. At a defined walking phase, the height of the raised tail is then measured. ${ }^{20} \mathrm{In}$ our study the wooden plate was $1500 \mathrm{~mm}$ long, $120 \mathrm{~mm}$ wide and $16 \mathrm{~mm}$ thick. All rats received training before injury until they could walk through the wooden plate smoothly. The defined walking phases were set at the time when the left hindlimb touched the surface of the bar while the right hindlimb was raised from the surface. All the activities of the rats were video recorded 1 day before and 1, 3, 5, 7 and 10 days after the SCI. Because the distance between rats and video camera was not exactly identical in different cases, the RHI was normalized by the thickness of the wooden plate. There is a variation in the size of the rats, and thus the standardized RHI (SRHI, SRHI $=\mathrm{RHI}_{\text {post-SCI }} / \mathrm{RHI}_{\text {pre-SCI }}$ ) was used for minimizing the variations of pre-injury RHI.

\section{Quantitation}

In our study, the method of densities and ratios of profile counts followed by calibration with the empirical method recommended by Coggeshall et al..$^{21,22}$ was applied. The $10 \mu \mathrm{m}$ thickness of the section ensures that the plane of central canal is cut through on every slide, rendering random selection of the slide possible. Randomly chosen slides and cell counting by an observer who was blinded to the experiment design were used.

Quantification of cells. For quantification of hemorrhage, the numbers of all of the RBCs in all the sections of the randomly chosen slide were counted. For quantification of the numbers of neurons, all of the Nissl-stained neurons in the rostral and caudal area of $600 \mu \mathrm{m}$ adjacent to lesion in all sections of the randomly chosen slide were counted. Then, calibration of the profile numbers was made by empirical method. Numbers of profiles per $\mathrm{mm}^{2}$ were considered as areal densities.

Quantification of immunofluorescence intensity. The immunofluorescence intensity of GFAP, Iba-1, RIP3 and MLKL was measured in the area of $400 \mu \mathrm{m}$ adjacent to the lesion center in all sections of the randomly chosen slide with ImagePro Plus Version 5.0 (Rockville, MD, USA). The relative immunofluorescence intensity was standardized by the corresponding area.

Quantification of the size of the lesion area. The boundary of lesion area is delineated by the GFAP-immunoreactive astrocytes. Four sections separated by $50 \mu \mathrm{m}$ lateral to the central canal were measured. The resulting areas were calculated by converting the pixels into millimeters using ImageJ.

\section{Statistical analysis}

All data are presented as mean \pm s.e. and analyzed with one-way analysis of variance, followed by Dunnett's post hoc test. $P<0.05$ was defined as statistically significant. ${ }^{*} P<0.05 ;{ }^{*} P<0.01$. 


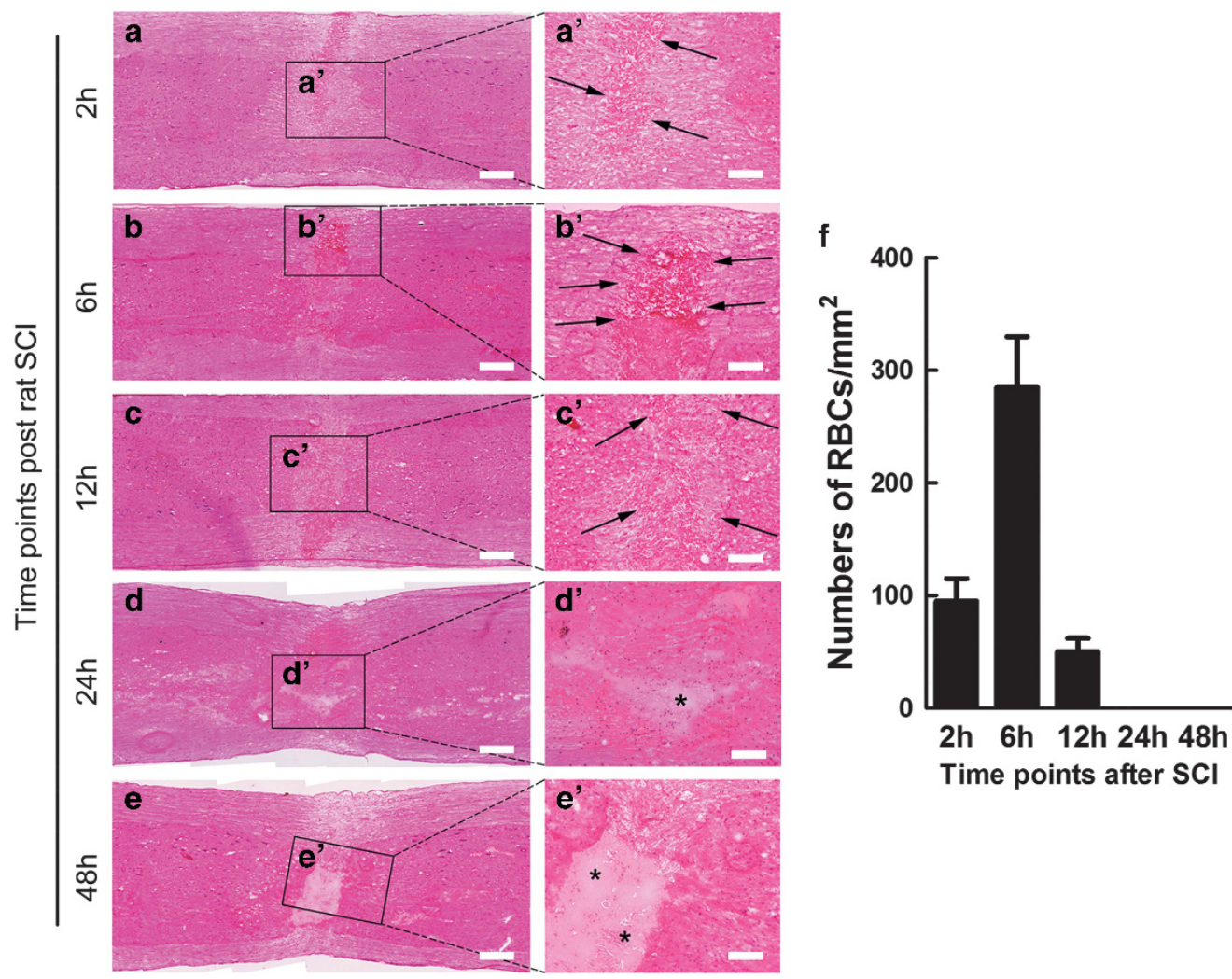

Figure 2 Duration of hemorrhage after SCC. (a-c) Numerous RBCs were observed at 2, 6 and $12 \mathrm{~h}$ after SCC. (d, e) None of RBCs were found at 24 and $48 \mathrm{~h}$ after SCC. Scale bars $=200 \mu \mathrm{m}$. (a'-e') Higher-magnification pictures from the white boxes in corresponding pictures. Arrows show RBCs and asterisks indicate the lesion center. Scale bars $=100 \mu \mathrm{m}$. (f) Quantification of the numbers of RBCs; $n=3$ per time point.

\section{RESULTS}

Duration of hemorrhage after SCC

HE staining illustrated that at 2, 6 and $12 \mathrm{~h}$ after SCC, the injured area of spinal cord was filled with RBCs (Figures 2aa'-cc'). No RBCs were observed at 24 and $48 \mathrm{~h}$ after SCC (Figures $2 \mathrm{~d}$ d' and ee').The data indicated that hemorrhage terminated before $24 \mathrm{~h}$ (Figure 2f). Based on this result Etamsylate was administrated right after SCI and at 5 and $10 \mathrm{~h}$. In all the following experiments the glucose control has no effect.

\section{Effects of Etamsylate on SCC}

Effects of Etamsylate on lesion area reduction. The effect of Etamsylate was initially evaluated by a dose-response experiment with concentrations of $0.025,0.05$ and $0.1 \mathrm{~g} \mathrm{~kg}^{-1}$. The data revealed that Etamsylate reduced the lesion size defined by the GFAP-immunoreactive boundary in a dose-dependent manner (Figures 3a-d). The group of $0.1 \mathrm{~g} \mathrm{~kg}^{-1}$ of Etamsylate reduced the lesion area by $40.91 \pm 5.71 \%$, whereas there were no significant differences between the other two lower dosages of Etamsylate-treated rats and the glucose-treated control ( $n=6$ rats/group, ${ }^{*} P<0.05$, Figure $3 \mathrm{e}$ ). As it had been shown in the above experiment that $0.1 \mathrm{~g} \mathrm{~kg}^{-1}$ of Etamsylate was effective, $0.1 \mathrm{~g} \mathrm{~kg}^{-1}$ only was used in the remaining studies.

Effects of Etamsylate on locomotion recovery. To evaluate locomotion at baseline (1 day before surgery) as well as 1, 3, 5, 7 and 10 days after SCC, BBB scoring and RHI assay were performed. The time course from 1 to 10 days post injury (d.p.i.) is an acute and intermediate phase for locomotion recovery of rats. ${ }^{19}$ Before surgery, all rats showed normal hindlimb movements with BBB score of 21 and SRHI value of 1. Compared with glucose control, the Etamsylate-treated rats showed significant higher BBB scores from 3 d.p.i. ( $n=6$ rats/group, ${ }^{*} P<0.05$, Figure 4a). Similarly, SRHI assay showed that the rump height was significantly elevated from 3 d.p.i. in Etamsylate-treated group ( $n=6$ rats/group, ${ }^{*} P<0.05$, Figure $4 \mathrm{~b}$ ).

Effects of Etamsylate on neuronal survival and necroptosis. The numbers of Nissl-stained neurons in the area of $600 \mu \mathrm{m}$ away from each side of the lesion were counted. The number of Nissl-stained neurons in Etamsylate-treated rats increased by 2.1 times ( $n=6$ rats/ group, ${ }^{\star} P<0.05$, Figures $\left.5 \mathrm{a}-\mathrm{c}\right)$.

Based on the results of our pilot experiment that the peak of necroptosis was found at 7 days after SCC in rats, the effects of early hemostasis were evaluated at the same time point after SCC. Necroptosis, which is a recently uncovered programmed necrosis of cells mediated by RIP3 and MLKL, can be identified by elevation of RIP3 and MLKL in the cells. ${ }^{23}$ To define the effects of Etamsylate on tissue necrosis, immunohistochemistry for RIP3 and MLKL was performed. Quantification in the areas $400 \mu \mathrm{m}$ rostral and dorsal to the lesion epicenter, as shown in Figure $5 \mathrm{~m}$, showed that the immunofluorescence intensity of RIP3 and MLKL was decreased by $41.47 \pm 2.16 \%$ and $50.32 \pm 3.84 \%$, respectively ( $n=3$ rats/group, ${ }^{*} P<0.05$, Figures $\left.5 \mathrm{~d}-\mathrm{g}\right)$. Western blots further showed that the expression of RIP3 and MLKL was reduced by $49.2 \pm 5.81 \%$ and $46.35 \pm 3.89 \%$, respectively $\left(n=3\right.$ rats/group, ${ }^{\star} P<0.05$, Figures $5 \mathrm{~h}-\mathrm{j})$. The mRNA of RIP3 and MLKL was decreased by $45.37 \pm 5.98 \%$ and $58.64 \pm 3.01 \%$, respectively $(n=3$ rats/group, ${ }^{\star} \mathrm{P}<0.05$, Figures $5 \mathrm{k}$ and $\left.\mathrm{l}\right)$. 

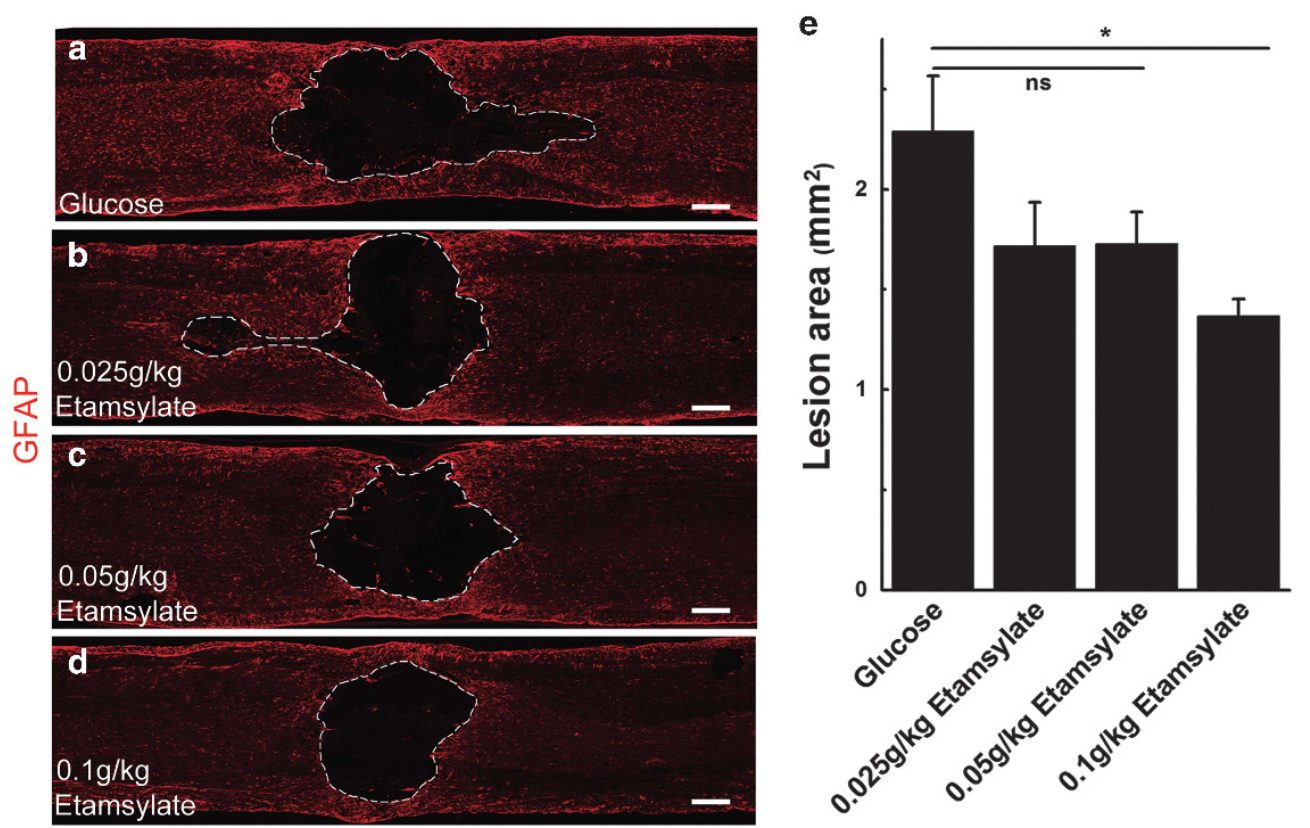

Figure $3 \mathrm{~A}$ dose-response experiment with different concentrations of Etamsylate. (a-d) Dashed lines indicate the lesion boundary defined by GFAPimmunoreactive astrocytic scar in glucose (a), $0.025 \mathrm{~g} \mathrm{~kg}^{-1}$ (b), $0.05 \mathrm{~g} \mathrm{~kg}^{-1}$ (c) and $0.1 \mathrm{~g} \mathrm{~kg}^{-1}$ groups (d). Scale bars $=300 \mu \mathrm{m}$. (e) Quantification of lesion area; $n=6$ /group. ${ }^{*} P<0.05$.

a

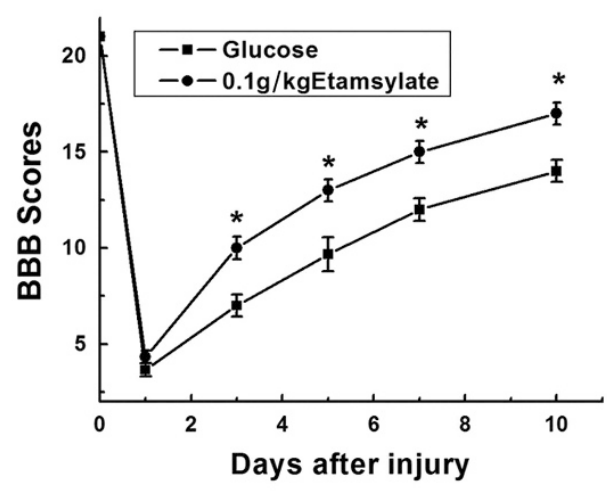

b

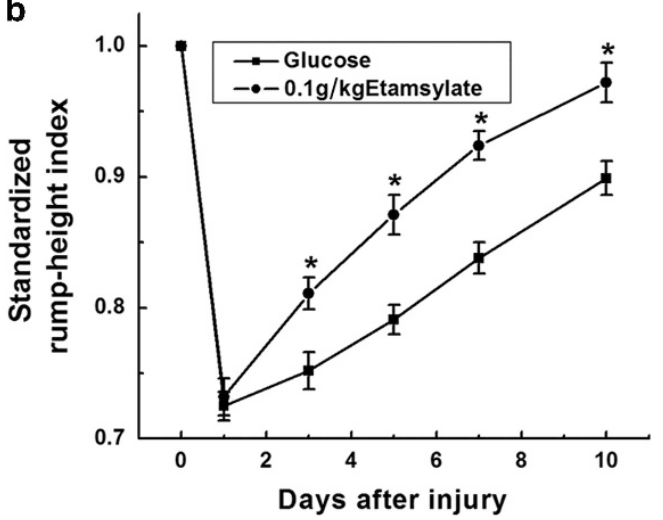

Figure 4 Locomotion improvement by Etamsylate. (a) BBB scores at 1, 3, 5, 7 and 10 days post SCC. The average BBB scores in Etamsylate-treated rats were significantly higher than those in control group at 3, 5, 7 and 10 days after injury. (b) SRHI values at 1, 3, 5, 7 and 10 days post SCC. Compared with the glucose control, the rump height was significantly elevated in Etamsylate-treated group at 3, 5, 7 and 10 days after injury; $n=6$ /group. ${ }^{*} P<0.05$.
Effects of Etamsylate on activation of astrocytes and microglia/macrophages. To assess the effects of Etamsylate on activation of astrocytes and microglia/macrophages, immunostainings for GFAP and Iba-1 were performed. Quantification in bilateral areas $400 \mu \mathrm{m}$ rostral and dorsal to lesion epicenter, as shown in Figure $5 \mathrm{~m}$, demonstrated that Etamsylate decreased the immunofluorescence intensity of GFAP and Iba- 1 by $52.14 \pm 4.25 \%$ and $44.11 \pm 5.03 \%$, respectively ( $n=6$ rats/ group, ${ }^{\star} P<0.05$, Figures $\left.6 \mathrm{a}-\mathrm{d}\right)$. The intensity of bands of GFAP and Iba-1 showed a reduction of $50.93 \pm 5.06 \%$ and $53.51 \pm 3.89 \%$ respectively in Etamsylate-treated rats $\left(n=3\right.$ rats/group, ${ }^{\star} P<0.05$, Figures $6 \mathrm{e}-\mathrm{h}$ ). The mRNA of GFAP and Iba-1 was decreased by $35.17 \pm 5.43 \%$ and $31.32 \pm 2.43 \%$ respectively in the Etamsylate group ( $n=3$ rats/group, ${ }^{*} P<0.05$, Figures $6 \mathrm{i}$ and $\mathrm{j}$ ).

Effects of Etamsylate on hemorrhage area and lesion area after SCT HE staining was performed to evaluate effects of Etamsylate on hemorrhage area $3 \mathrm{~h}$ after SCT. Numerous RBCs were found surrounding the transection site in glucose control (Figure 7a). The hemorrhage area showed a reduction of $71.38 \pm 7.12 \%$ ( $n=5$ /group, ${ }^{*} P<0.01$, Figures $7 \mathrm{~b}$ and $\mathrm{c}$ ). Immunostaining of GFAP was performed to mark the boundary of lesion 10 days after SCT to assess the effects of Etamsylate on the size of the lesion area. The data showed that the lesion area was reduced by $42.11 \pm 3.19 \%$ ( $n=5$ /group, ${ }^{\star} P<0.05$, Figures $\left.7 \mathrm{~d}-\mathrm{f}\right)$.

\section{DISCUSSION}

SCI was regarded as 'A disease that cannot be treated' in Egypt more than 3500 years ago. Several millennia have passed, and physicians were still unable to treat injuries to the spinal cord. Only in the middle of the twentieth century did medicine learn to keep spinally injured people alive. Individuals paralyzed by trauma to the spinal cord numbered 250000 in the United States alone and an estimated 2 million worldwide. ${ }^{24}$ In the Western Han dynasty of ancient China (206 BC to $24 \mathrm{AD}$ ) a stone relief showed a dear shot by an arrow at mid thoracic vertebra with its lower legs paralyzed. 

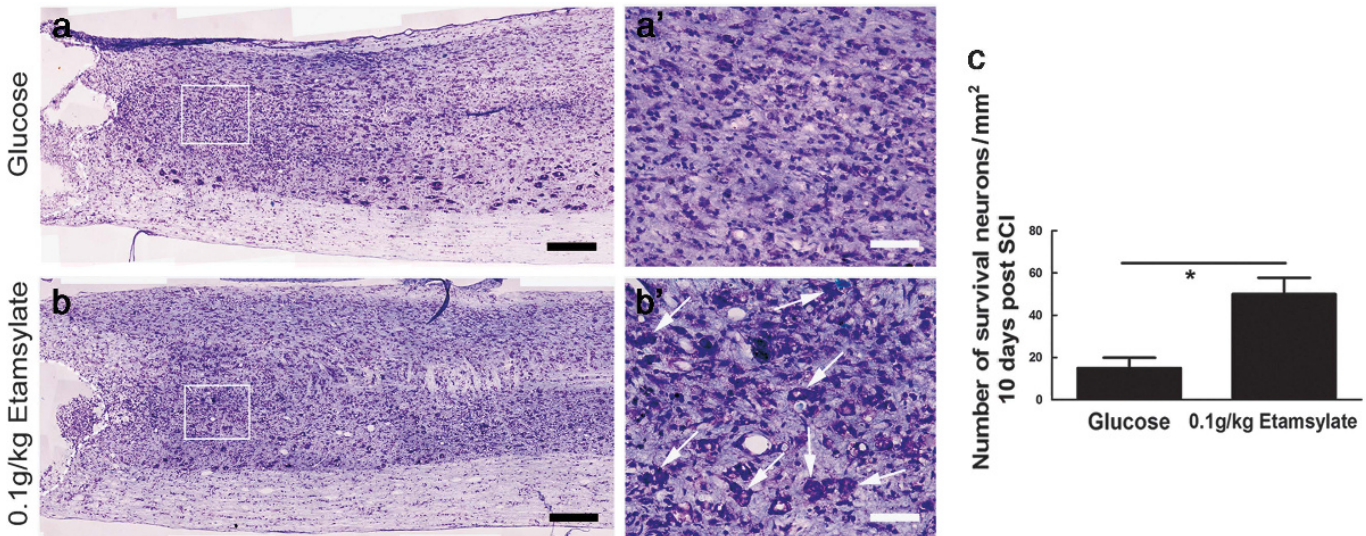
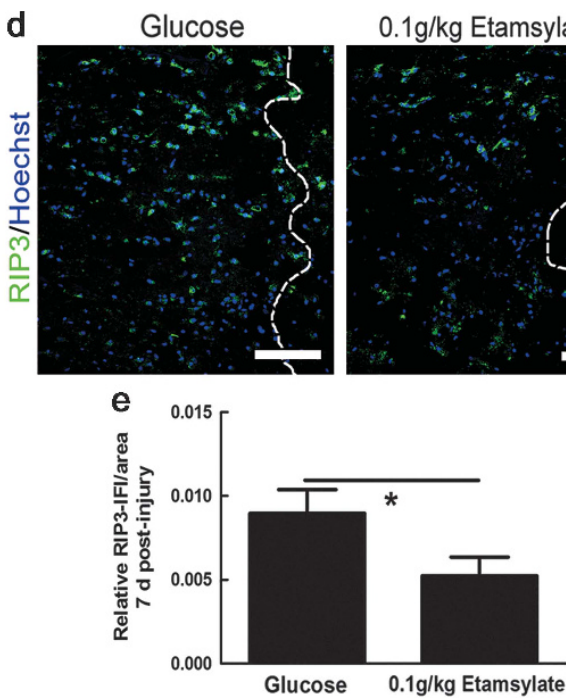
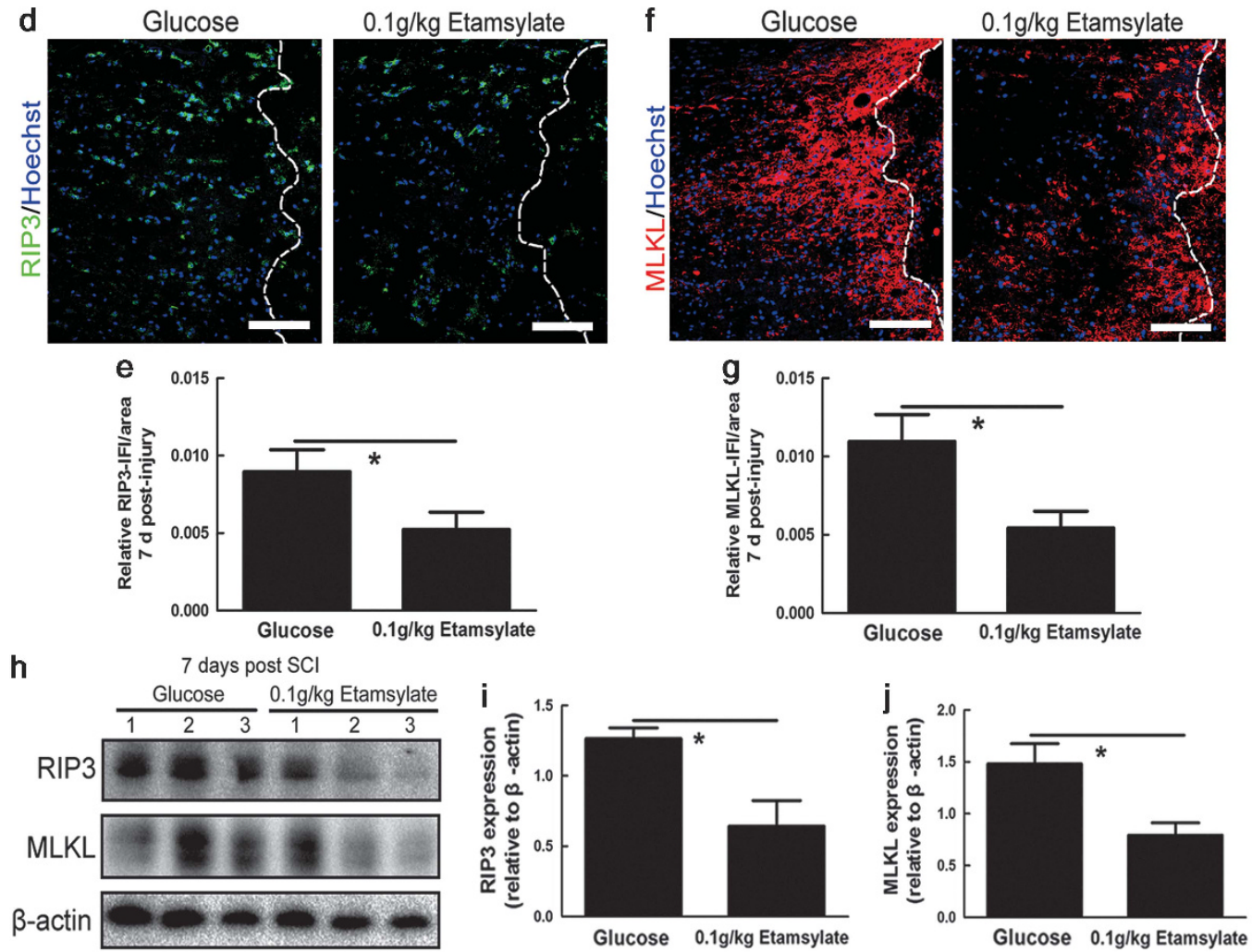
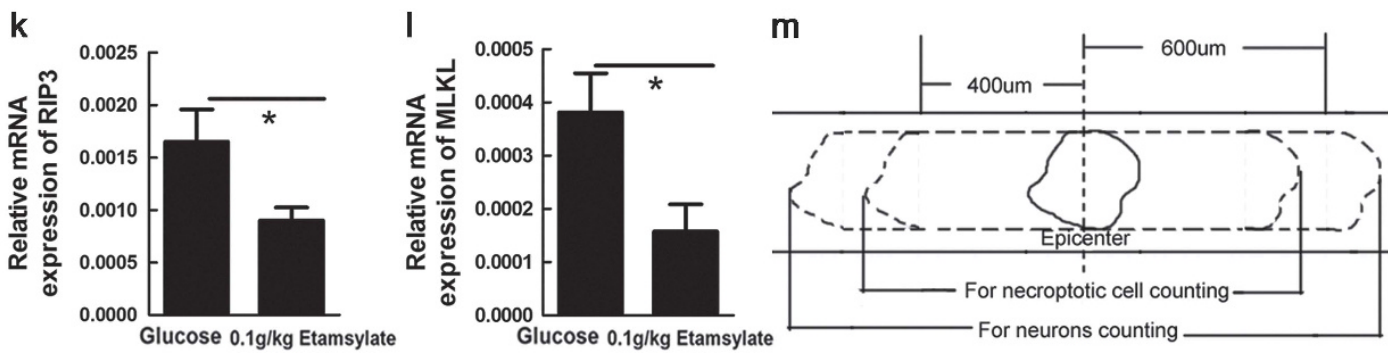

Figure 5 Survival of neurons shown by Nissl staining at 10 days after SCC and necrosis reduction by Etamsylate at 7 days after SCC. (a, b) A representative section of the glucose control (a) and Etamsylate-treated rats (b). Broken lines mark the lesion borders. Note the abundance of inflammatory cells in the glucose control and neurons in Etamsylate-treated group. Scale bars $=200 \mu \mathrm{m}$. (a', b') Higher-magnification pictures from the boxes in (a) and (b). Arrows indicate Nissl-stained neurons. Scale bars $=100 \mu \mathrm{m}$. (c) Quantification of the numbers of Nissl-stained neurons; $n=6 / g r o u p . ~ * P<0.05$. (d, f) Immunohistochemistry for RIP3 and MLKL, two important molecules underlying necroptosis. Scale bars $=100 \mu \mathrm{m}$. (e, g) Quantification of the immunofluorescence intensity (IFI)/area of RIP3 and MLKL; $n=3$ /group. ${ }^{*} P<0.05$. (h) Western blotting of RIP3 and MLKL, and $\beta$-actin was used as a loading control. (i, j) The quantified data of the expression of RIP3 and MLKL; $n=3 /$ group. ${ }^{*} P<0.05$. (k, I) Quantification of mRNA levels of RIP3 and MLKL; $n=3$ /group. ${ }^{*} P<0.05$. (m) A chart showing the area where the cells were counted. 

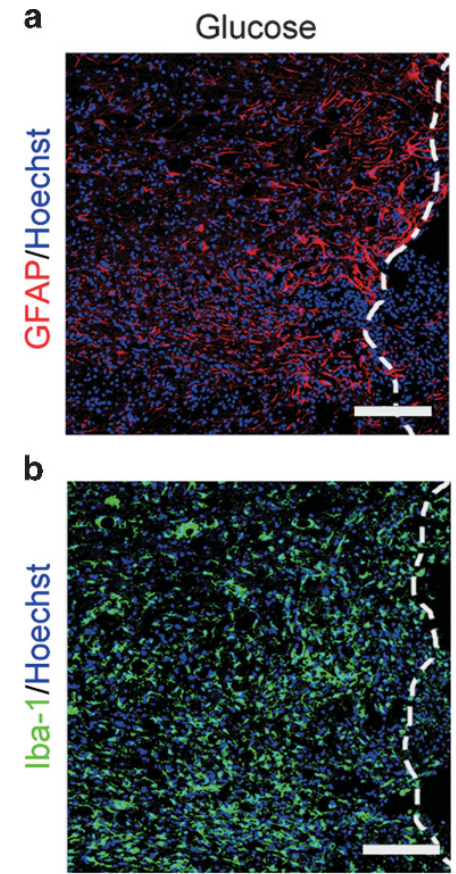

e

10 days post $\mathrm{SCl}$

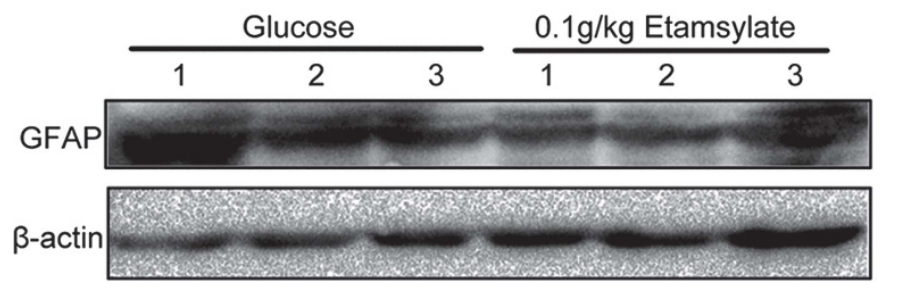

f
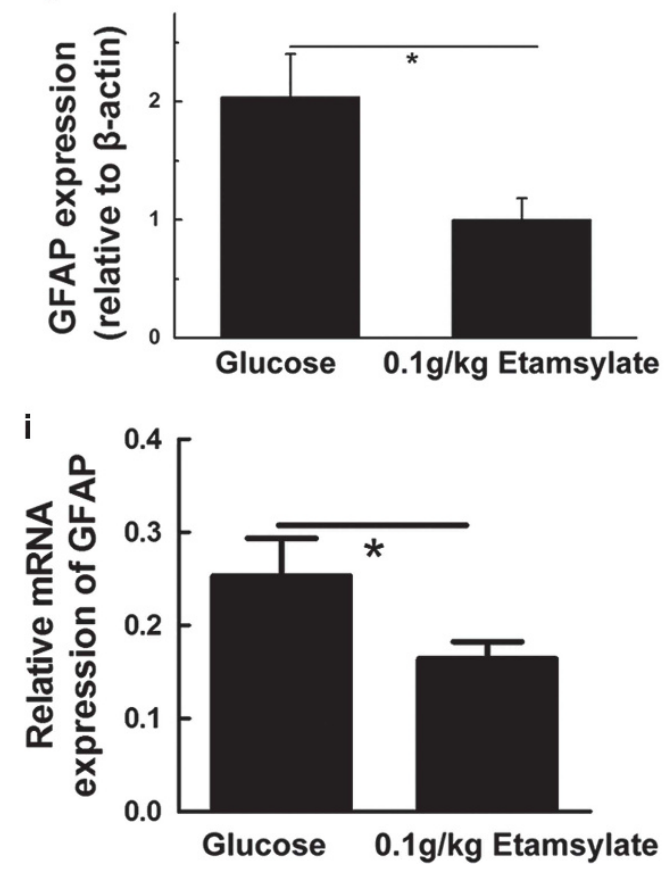
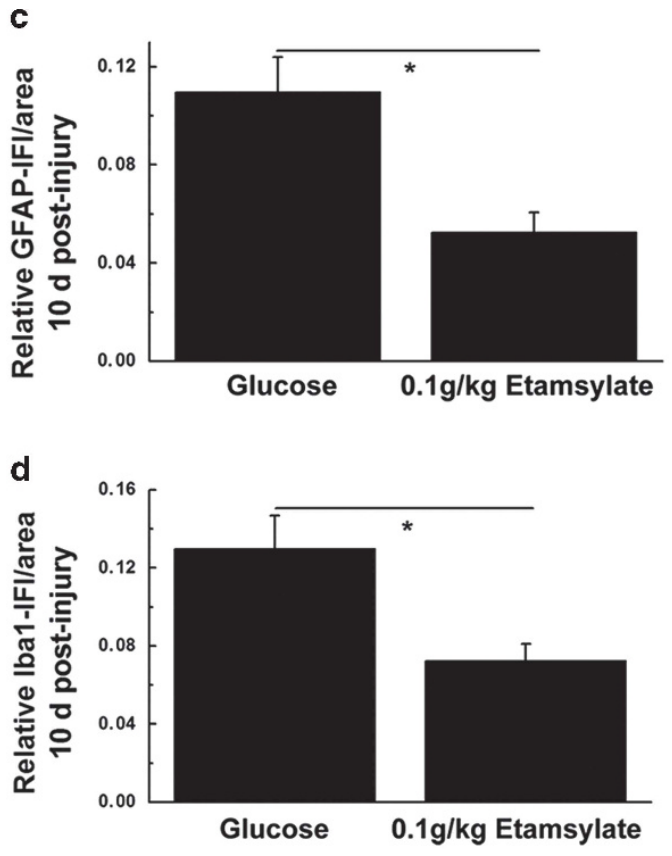

g

10 days post $\mathrm{SCl}$
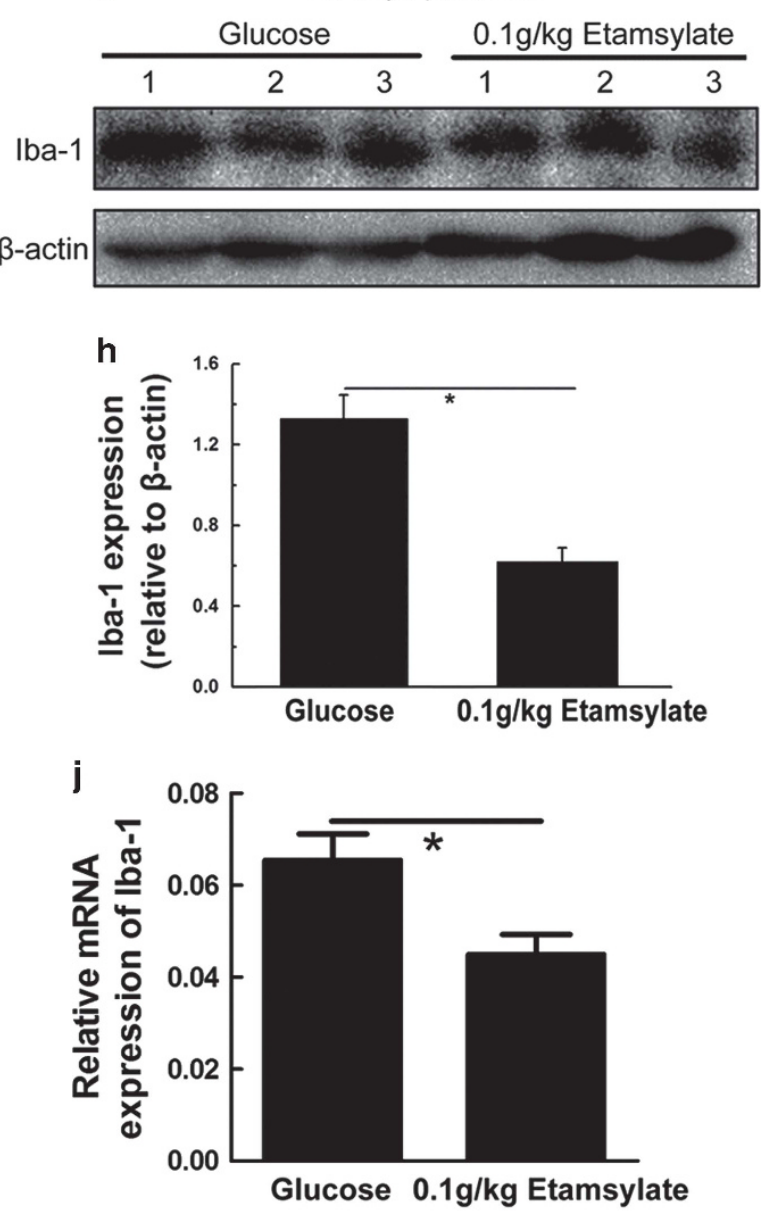

Figure 6 Effects of Etamsylate on GFAP and Iba-1expression at 10 days after SCC. (a, b) GFAP and Iba-1 immunoreactivity in glucose control (a) and Etamsylate-treated group (b). (c, d) Quantification of the immunofluorescence intensity (IFI)/area of GFAP and Iba- 1 . Scale bars $=100 \mu \mathrm{m}$; $n=6 / g r o u p$. ${ }^{*} P<0.05$. (e, g) Western blots were probed for GFAP and Iba-1, and $\beta$-actin was used as a loading control. (f, $\left.\mathbf{h}\right)$ Quantification of the expression of GFAP and Iba-1; $n=3$ /group. ${ }^{*} P<0.05$. (i, j) Quantification of mRNA levels of GFAP and Iba- $1 ; n=3$ /group. ${ }^{*} P<0.05$. 

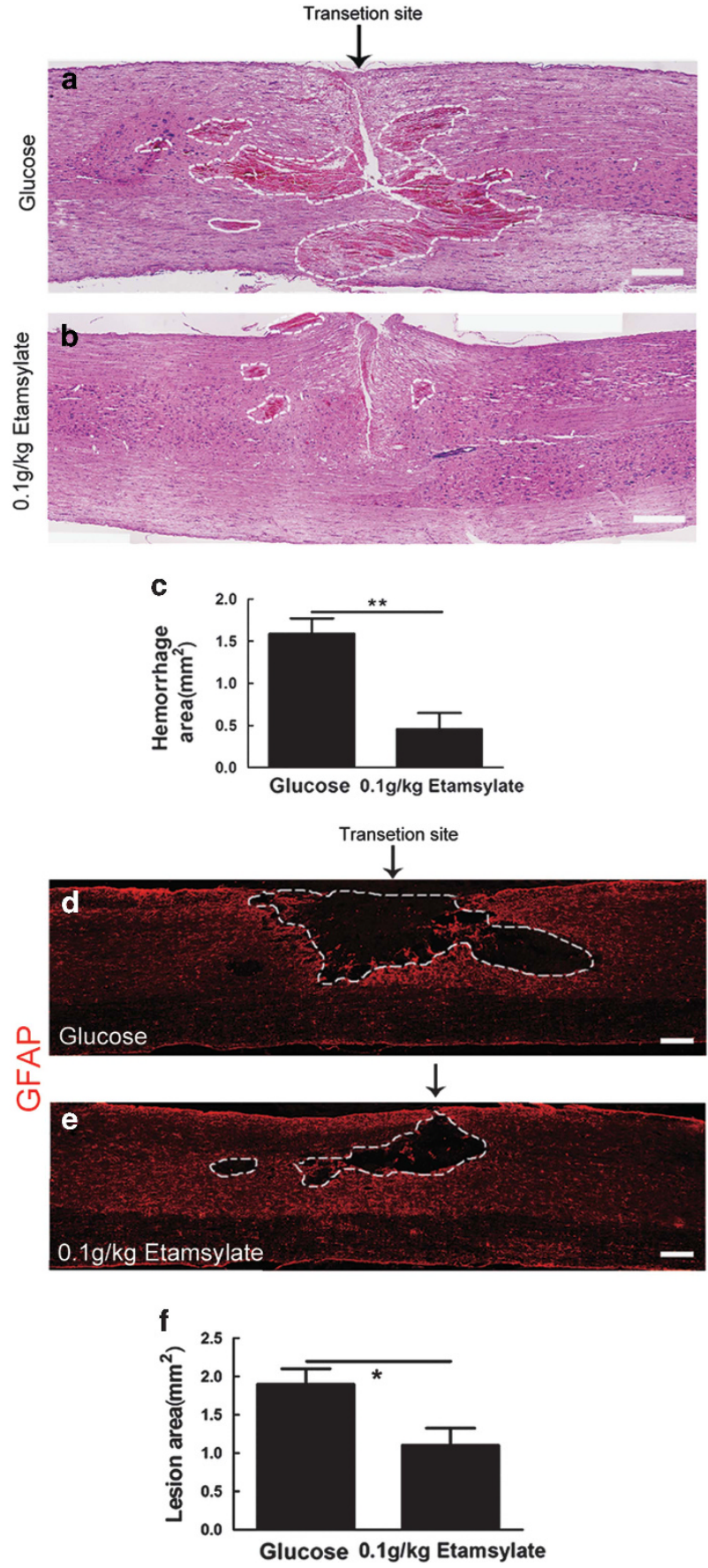

Figure 7 Reduction of hemorrhage and area of lesion cavity by Etamsylate after SCT. (a, b) Hemorrhage area in glucose control (a) and Etamsylate group (b). The arrow showed the transection site. Scale bars $=400 \mu \mathrm{m}$. (c) Quantification of the hemorrhage area; $n=5$ /group. ${ }^{* *} P<0.01$. (d, e) Dashed lines indicate the lesion boundary defined by GFAPimmunoreactive astrocytes in glucose control (d) and Etamsylate group (e). Scale bars $=300 \mu \mathrm{m}$. (f) Quantification of lesion area; $n=5 /$ group. ${ }^{*} P<0.05$.

The incidence and prevalence of SCI has been estimated in China, but it does not have any value, because there are no data at all in remote areas in China. Nevertheless, there is no doubt that thousands of people in China are suffering from SCI, and a large number added each year, because the causes of SCI are very common in China. The life expectancy of the patients in wheelchairs is about the same in healthy people, and thus the pain of the patients and their families will last for years, aside from the burden on the society. Therefore, the study of SCI is one of the major focuses of medical research the world over.

Bleeding starts at the very moment the spinal cord is injured, either by contusion or transection, and lasts for a certain period of time, resulting in a variety of pathological changes in the cord. In a couple of days, the damaged tissue, mainly necrotic, will be encircled by astrocytic scar that gradually expands till it reaches the final irreversible stage. ${ }^{25}$ Therefore, the logical treatment of spinal contusion should be to remove the necrotic substance in the astrocytic cavity (debridement), thereby terminating the process of the secondary injury. Our group has previously realized the importance of early treatment for SCI and shown that surgical debridement of the injury site between 4 and 14 days after SCI resulted in significant functional improvements. ${ }^{26}$

Although ischemia occurs in SCC, ${ }^{27}$ ischemic stroke is different from hemorrhagic stroke, and the latter has similar mechanism of the spread of secondary degeneration in SCI. ${ }^{28,29}$ It has been reported that hemostatic drugs such as tranexamic acid and activated recombinant factor VII can arrest ongoing bleeding and minimize hematoma growth on intracerebral hemorrhage. ${ }^{30,31}$ It supports our result of beneficial effects of early hemostasis that can effectively reduce the lesion area and tissue necrosis, enhance neuronal survival, alleviate the activation of microglia and astrocytes and facilitate functional recovery after SCC in rats. In SCT, hemostasis decreased hemorrhage area and lesion area. It has been reported that fibrinogen leaked from the blood vessels in hemorrhagic condition can activate astrocytes and microglia, and the latter are shown to have detrimental effects on regeneration in the central nervous system. ${ }^{32,33}$ Our results of the beneficial effects of hemostasis could well be partially due to this effect.

The present study has demonstrated that early hemostasis is beneficial for SCI in the rat. As there is no harm done in hemostasis, it can be easily translated into clinical practice, particularly in an ambulance. On this basis, the result of 'Early surgical intervention of spinal cord contusion' can be much improved.

In conclusion, the present study demonstrated that early hemostasis has significant beneficial effects on SCI in the rat that has potential to be translated into clinical practice.

\section{CONFLICT OF INTEREST}

The authors declare no conflict of interest.

\section{ACKNOWLEDGEMENTS}

This work was supported by grants from the National Natural Science Foundation of China (NSFC, 81371364) to Professor Gong Ju, NSFC (31271583) to Dr Ya-Zhou Wang and research fellowship from Fourth Military Medical University (2013D09) to Dr Hong Fan. We appreciate the technical assistances by Hai-Feng Zhang.

1 Liu JM, Long XH, Zhou Y, Peng HW, Liu ZL, Huang SH. Is urgent decompression superior to delayed surgery for traumatic spinal cord injury? A meta-analysis. World Neurosurg 2016; 87: 124-131.

2 Mautes AE, Weinzierl MR, Donovan F, Noble LJ. Vascular events after spinal cord injury: contribution to secondary pathogenesis. Phys Ther 2000; 80: 673-687.

3 Losey P, Anthony DC. Impact of vasculature damage on the outcome of spinal cord injury: a novel collagenase-induced model may give new insights into the mechanisms involved. Neural Regen Res 2014; 9: 1783-1786.

4 Profyris C, Cheema SS, Zang D, Azari MF, Boyle K, Petratos S. Degenerative and regenerative mechanisms governing spinal cord injury. Neurobiol Dis 2004; 15: 415-436.

5 Losey P, Young C, Krimholtz E, Bordet R, Anthony DC. The role of hemorrhage following spinal-cord injury. Brain Res 2014; 1569: 9-18. 
6 Liu X, Su H, Chu TH, Guo A, Wu W. Minocycline inhibited the pro-apoptotic effect of microglia on neural progenitor cells and protected their neuronal differentiation in vitro. Neurosci Lett 2013; 542: 30-36.

7 Liu X, Chu TH, Su H, Guo A, Wu W. Neural progenitor cell apoptosis and differentiation were affected by activated microglia in spinal cord slice culture. Neurol Sci 2014; 35: 415-419.

8 Lee JY, Choi HY, Yune TY. MMP-3 secreted from endothelial cells of blood vessels after spinal cord injury activates microglia, leading to oligodendrocyte cell death. Neurobiol Dis 2015; 82: 141-151.

9 Kroner A, Greenhalgh AD, Zarruk JG, Passos Dos Santos R, Gaestel M, David S. TNF and increased intracellular iron alter macrophage polarization to a detrimental M1 phenotype in the injured spinal cord. Neuron 2014; 83: 1098-1116.

10 Lammertse D, Dungan D, Dreisbach J, Falci S, Flanders A, Marino R et al. Neuroimaging in traumatic spinal cord injury: an evidence-based review for clinical practice and research. J Spinal Cord Med 2007; 30: 205-214.

11 Shepard MJ, Bracken MB. Magnetic resonance imaging and neurological recovery in acute spinal cord injury: observations from the National Acute Spinal Cord Injury Study 3. Spinal Cord 1999; 37: 833-837.

12 Garay RP, Chiavaroli C, Hannaert P. Therapeutic efficacy and mechanism of action of ethamsylate, a long-standing hemostatic agent. Am J Ther 2006; 13: 236-247.

13 Alvarez-Guerra M, Hernandez MR, Escolar G, Chiavaroli C, Garay RP, Hannaert P. The hemostatic agent ethamsylate enhances P-selectin membrane expression in human platelets and cultured endothelial cells. Thromb Res 2002; 107: 329-335.

14 Fraser IS, Porte RJ, Kouides PA, Lukes AS. A benefit-risk review of systemic haemostatic agents: part 1: in major surgery. Drug Saf 2008; 31: 217-230.

15 Lewis GJ. Does ethamsylate increase the incidence of venous thrombosis? Br Med J 1984; 288: 899-900.

16 Rennie JM, Lam PK. Effects of ethamsylate on cerebral blood flow velocity in premature babies. Arch Dis Child 1989; 64: 46-47.

17 Plemel JR, Duncan G, Chen KW, Shannon C, Park S, Sparling JS et al. A graded forceps crush spinal cord injury model in mice. J Neurotrauma 2008; 25: 350-370.

18 Fan H, Liu X, Tang HB, Xiao P, Wang YZ, Ju G. Protective effects of Batroxobin on spinal cord injury in rats. Neurosci Bull 2013; 29: 501-508.

19 Basso DM, Beattie MS, Bresnahan JC. A sensitive and reliable locomotor rating scale for open field testing in rats. J Neurotrauma 1995; 12: 1-21.

20 Sedy J, Urdzikova L, Jendelova P, Sykova E. Methods for behavioral testing of spinal cord injured rats. Neurosci Biobehav Rev 2008; 32: 550-580.

21 Coggeshall RE, Lekan HA. Methods for determining numbers of cells and synapses: a case for more uniform standards of review. J Comp Neurol 1996; 364: 6-15.

22 Coggeshall RE, La Forte R, Klein CM. Calibration of methods for determining numbers of dorsal root ganglion cells. J Neurosci Methods 1990; 35: 187-194.
23 Sun L, Wang H, Wang Z, He S, Chen S, Liao D et al. Mixed lineage kinase domain-like protein mediates necrosis signaling downstream of RIP3 kinase. Cell 2012; 148: 213-227.

24 Vikhanski L. In Search of the Lost Cord: Solving the Mystery of Spinal Cord Regeneration. Joseph Henry Press: Washington, DC, USA. 2001.

25 Kalb RG, Strittmatter SM. Neurobiology of Spinal Cord Injury. Humana Press: Totowa, NJ, USA. 2000

26 Zhu H, Feng YP, Young W, You SW, Shen XF, Liu YS et al. Early neurosurgical intervention of spinal cord contusion: an analysis of 30 cases. Chin Med J 2008; 121: 2473-2478.

27 Shen XF, Zhao Y, Zhang YK, Jia LY, Ju G. A modified ferric tannate method for visualizing a blood vessel and its usage in the study of spinal cord injury. Spinal Cord 2009; 47: 852-856.

28 Lopez MS, Dempsey RJ, Vemuganti R. Resveratrol neuroprotection in stroke and traumatic CNS injury. Neurochem Int 2015; 89: 75-82.

29 Chen HY, Lin JM, Lin CC. Raffinee, a free radical scavenger, in the treatment of subacute stage brain and spinal cord lesions: a case report. Am J Chin Med 1998; 26: 97-108.

30 Mayer SA. Ultra-early hemostatic therapy for intracerebral hemorrhage. Stroke 2003; 34: 224-229.

31 Wartenberg KE, Mayer SA. Ultra-early hemostatic therapy for intracerebral hemorrhage: future directions. Front Neurol Neurosci 2015; 37: 107-129.

32 Schachtrup C, Ryu JK, Helmrick MJ, Vagena E, Galanakis DK, Degen JL et al. Fibrinogen triggers astrocyte scar formation by promoting the availability of active TGFbeta after vascular damage. J Neurosci 2010; 30: 5843-5854.

33 Davalos D, Ryu JK, Merlini M, Baeten KM, Le Moan N, Petersen MA et al. Fibrinogeninduced perivascular microglial clustering is required for the development of axonal damage in neuroinflammation. Nat Commun 2012; 3: 1227.

(1)(2) This work is licensed under a Creative Commons Attribution-NonCommercial-ShareAlike

4.0

International License. The images or other third party material in this article are included in the article's Creative Commons license, unless indicated otherwise in the credit line; if the material is not included under the Creative Commons license, users will need to obtain permission from the license holder to reproduce the material. To view a copy of this license, visit http://creativecommons.org/ licenses/by-nc-sa/4.0/ 